\title{
Evaluation and management of IgA nephropathy
}

\author{
CK Cheung, JKF Boyd and J Feehally
}

\section{Introduction}

Immunoglobulin A (IgA) nephropathy (IgAN) is the most common pattern of glomerulonephritis seen worldwide, and is an important cause of progressive chronic kidney disease, with some $20 \%$ of patients developing end-stage renal disease within 20 years of diagnosis. It is defined by renal histology showing predominant glomerular IgA deposition. It was identified as a separate entity during the 1960 s, when immunofluorescence to detect immunoglobulin and complement deposition was first being applied to renal biopsies.

\section{Is IgA nephropathy a single disease?}

\section{Clinical features}

Although IgAN can present at any age, the peak incidence is between the second and fourth decades. The commonest presentation, seen in up to $40 \%$ of patients, is of visible haematuria occurring within 12-72 hours of a mucosal infection, typically an upper respiratory tract infection (sometimes referred to as 'synpharyngitic haematuria'). The next most common presentation is the detection of asymptomatic urinary abnormalities, non-visible haematuria with or without proteinuria, for example on employment or life insurance medical screening. Others will already have more advanced chronic kidney disease at presentation with hypertension, heavy proteinuria and falling glomerular filtration rate (GFR). Approximately $5 \%$ of patients will present with nephrotic syndrome. Although the clinical course is usually slowly progressive, patients can present, albeit rarely, with rapidly progressive renal failure, or with accelerated hypertension.

IgAN is also less commonly seen secondary to other conditions, including chronic liver disease (typically alcoholic cirrhosis), coeliac disease, ankylosing spondylitis, Reiter's syndrome and dermatitis herpetiformis.

When IgAN is accompanied by an IgA-mediated small vessel systemic vasculitis (typically affecting skin, joints and gut), it is known as Henoch-Schönlein purpura (HSP). HSP can also occur without renal involvement. In addition, although it can occur at any age, it predominantly affects young children, for as yet unknown reasons.

CK Cheung, ${ }^{1,2}$ SpR in nephrology; JKF Boyd, ${ }^{1,2}$ academic clinical fellow in nephrology; and J Feehally, ${ }^{1,2}$ consultant nephrologist and professor of renal medicine

${ }^{1}$ The John Walls Renal Unit, University Hospitals of Leicester, UK; ${ }^{2}$ Department of Infection, Inflammation \& Immunity, University of Leicester, UK
There is marked geographical variation in IgAN, with the highest incidence reported in the Pacific Rim, and much lower incidences in Africa compared with Europe. There is a marked male predominance in most populations, but it is equally common among women in East Asia. These variations might be in part explicable by differing approaches to renal biopsy; that is, IgAN will be diagnosed more where there is a low threshold to perform a renal biopsy for minor urinary abnormalities. However, it also remains probable that there is a genetic basis (as yet undefined) for some of this variability.

\section{Pathology}

IgA deposits are always mesangial and mainly of the IgA1 isotype. Mesangial IgA is detected on renal biopsy by immunofluorescence or immunoperoxidase staining. There can also be IgG, IgM and C3 deposits, but IgA is always the most prominent. There can also be capillary loop as well as mesangial IgA deposition, which is probably associated with a worse prognosis. Mesangial electron-dense deposits corresponding to the IgA positivity are seen on electron microscopy. The glomerular IgA deposition is accompanied by highly variable light microscopic appearances, which do not correlate with the extent of IgA deposition. The glomeruli might appear normal, but diffuse or focal mesangial hypercellularity and extracellular matrix expansion are most common. With advancing injury, the features do not differ from those seen in other progressive forms of chronic glomerulonephritis, such as glomerulosclerosis and tubular atrophy with interstitial fibrosis. A kidney biopsy taken during episodes of visible haematuria might show only acute tubular injury owing to heavy haematuria, or severe glomerular inflammation with crescent formation.

\section{Recurrence after renal transplantation}

Although recurrence of IgAN after kidney transplantation is common, it is not inevitable. ${ }^{1}$ Mesangial IgA deposition can be detected in more than $50 \%$ of transplants by 5 years, but only $5 \%$ will have graft failure attributable to that recurrence. There is no evidence that any particular transplant immunosuppressive regimen prevents recurrence of IgAN.

\section{Pathogenesis}

An important pathogenic mechanism in many patients with IgAN seems to be the mesangial deposition of IgA1 with characteristic aberrant glycosylation. ${ }^{2}$ However, this abnormality, which is the focus of much recent research, seems to be 'neither necessary nor sufficient' to provoke IgAN. At least $10 \%$ of 
patients with IgAN have no detectable abnormality of IgA glycosylation. The roles of any additional pathogenic elements, for example genetic or environmental, in the processes that follow IgAl deposition provoking glomerular inflammation and renal scarring are not well defined. Despite the close relation between mucosal infections and macroscopic haematuria in many patients, there is no evidence of a role for any specific antigen, although the formation of IgA1-containing circulating immune complexes appears important.

Most cases of IgAN are sporadic, although familial cases do occur. Different risk loci have been identified in several welldefined kindreds with high IgAN penetrance. However, genetic studies in sporadic cases have been unrewarding so far and have not yielded strong genetic associations, even though IgA glycosylation appears to be a heritable trait.

\section{A single disease?}

Why are the clinical and pathological features associated with IgAN so variable? Why do the incidence and gender variation differ in different parts of the world? Why is recurrence of IgAN after transplantation not inevitable? Why has no single pathogenic mechanism yet emerged? Why have genetic studies not yielded strong risk associations? This marked heterogeneity means that there is currently insufficient evidence to regard IgAN as a single disease or even the same disease in all parts of the world. These marked variations suggest that a single pathogenic mechanism does not account for either IgA deposition or its effects on promoting renal failure and clinical disease manifestations.

\section{Can IgAN be diagnosed without a kidney biopsy?}

Post-infectious (most commonly post-streptococcal) glomerulonephritis can present with visible haematuria, but can usually be distinguished from IgAN because there is typically a 2-4-week delay between the streptococcal infection and visible haematuria. By contrast, in IgAN, haematuria occurs within 12-24 hours. However, other glomerular diseases can also present with episodes of visible haematuria, especially in young adults. Although there has been interest in non-invasive tests, a high serum IgA level is not diagnostic. There has been recent interest in the measurement of altered glycosylation of serum IgAl, but this is not a universal finding in IgAN and lacks the sensitivity and specificity to be a clinically useful diagnostic test. Therefore, IgAN remains a histopathological definition, and the diagnosis can only be made by renal biopsy.

In those patients who present with isolated non-visible haematuria (ie without proteinuria and with preserved GFR), a renal biopsy is not usually indicated as the histological diagnosis rarely changes management. However, urological causes of haematuria must always be excluded by appropriate investigation. Nevertheless, if renal biopsy is not performed, a significant number of low-grade cases of IgAN will go undetected and regular follow-up is recommended because of a small but definite risk of later progressive renal disease. However, IgAN is not the only glomerular disease causing isolated non-visible haematuria; therefore, a presumptive diagnosis of IgAN should never be made on clinical grounds in the absence of a renal biopsy.

\section{Is it possible to predict which patients with IgAN will develop kidney failure?}

Proteinuria is the most important clinical predictor of progressive IgAN. Not only proteinuria at presentation, but also time-averaged proteinuria during prolonged follow-up are predictive of outcome. $^{3}$ Those patients in whom proteinuria remains $<1 \mathrm{~g} / \mathrm{d}$ (whether spontaneously or in response to therapy) have an excellent prognosis. Tight blood pressure control is also critical to minimising risk of progression. ${ }^{4}$ Those with continuing heavy proteinuria and uncontrolled blood pressure have the highest risk of progression. ${ }^{5}$

Episodes of visible haematuria become less frequent over time, although most patients will have persistent non-visible haematuria. Perhaps unexpectedly, those with episodes of visible haematuria appear to have a better prognosis than those without. This might well be because of lead-time bias, with episodic visible haematuria resulting in earlier presentation and, therefore, extending the perceived duration of the clinical course.

Until recently, pathological features in IgAN were not regarded as reliably predictive of outcome. Indeed, it might perhaps not be expected that pathological features in the initial diagnostic biopsy could inform prognosis, given the prognostic value that can be obtained from clinical information (ie blood pressure and proteinuria) at presentation and obtained during long-term followup. No pathological classification for IgAN had proved universally reliable in routine clinical practice, until the recent Oxford Classification of IgAN, which has now identified pathological predictors of outcome in IgAN. ${ }^{6}$ Importantly, this classification was derived from a study in which only those features that could reliably and reproducibly be identified by a group of pathologists were included. The four variables are: mesangial cell proliferation; endocapillary proliferation; segmental glomerulosclerosis; and tubulointerstitial scarring. Each of these is simple to score in routine clinical practice and is independently associated with the risk of developing progressive renal failure. Furthermore, each of these features remains predictive even if clinical information at presentation and during follow-up is known. Although further validation of this classification is needed, it has the potential to refine prognosis for individual patients, and also to narrow recruitment to clinical trials of new treatments for IgAN, by facilitating identification of those at highest risk and, therefore, most likely to benefit from an intervention.

\section{How good is the evidence to guide treatment of IgAN?}

As yet, there are no specific therapies for IgAN to interrupt the deposition of IgA, so available therapies are aimed at the inflammatory and profibrotic events that follow IgA deposition. These 
therapies are probably generic and could be applicable to other slowly progressive glomerular diseases. Development of evidencebased treatment strategies in IgAN has been hindered by the heterogeneity seen in clinical presentation and disease course, and the lengthy duration of studies needed to demonstrate changes in hard endpoints, such as the risk of renal failure, rather than surrogate endpoints, such as a reduction in proteinuria. Few studies have been designed with an adequate 'run-in' phase in which blood pressure control and renin-angiotensin system (RAS) blockade have been adequately achieved before immunosuppressive interventions are tested, although newer studies now underway are better designed. ${ }^{7}$ An internationally accepted clinical practice guideline for glomerulonephritis has recently critically reviewed available evidence for the treatment of IgAN. ${ }^{8}$ Current treatment recommendations are summarised in Table 1.

\section{Proteinuria and blood pressure}

Strict blood pressure control is important in preserving renal function in IgAN as in other forms of proteinuric chronic kidney disease: there is good evidence to support a goal of a blood pressure of $130 / 80 \mathrm{mmHg}$ when proteinuria is below $1 \mathrm{~g} / \mathrm{d}$ and below 125/75 when proteinuria is over $1 \mathrm{~g} / \mathrm{d}$.

Proteinuria $>1 \mathrm{~g} / \mathrm{d}$ in IgAN is associated with a worse renal outcome, with higher levels being associated with a more rapid decline in renal function. Starting an angiotensin-converting enzyme inhibitor (ACEi) or angiotensin receptor blocker (ARB) is recommended in those patients with proteinuria $>1 \mathrm{~g} / \mathrm{d}$ with the dose titration to achieve proteinuria below $1 \mathrm{~g} / \mathrm{d}$. Evidence is less robust to justify use of these agents with proteinuria $0.5-1.0 \mathrm{~g} / \mathrm{d}$. Salt restriction is crucial to the efficacy of RAS blockade in reducing proteinuria, as well as in optimising blood pressure control.

\section{Corticosteroids}

Available evidence favours the use of corticosteroids in those patients with proteinuria $>1 \mathrm{~g} / \mathrm{d}$ and GFR $>50 \mathrm{ml} / \mathrm{min}$; how- ever, this is limited to high-dose regimens (typically for 6 months), and has not yet ideally tested the value of this approach when there is adequate RAS blockade. It should also be noted that those patients with nephrotic-range proteinuria were excluded from these studies. The obvious concern with this strategy is the short- and long-term effects of such highdose corticosteroid use. This remains controversial, but many experts do recommend a trial of corticosteroids if maximal RAS blockade and proper blood pressure control have failed to improve proteinuria.

\section{Other immunosuppressive agents: cyclophosphamide, azathioprine and mycophenolate mofetil}

There is no convincing evidence to support the use of cyclophosphamide or azathioprine in IgAN. Mycophenolate mofetil has more recently been tested in two pilot random clinical trials (RCTs), one in Hong Kong and one in Europe, in patients with IgAN and significant proteinuria. The results are variable: only the study from Hong Kong showed a benefit in terms of a significant reduction in renal failure at 6 years. Additional studies with sufficient power are needed to resolve the role of mycophenolate mofetil, but the disparity between the initial data from Hong Kong and Europe does suggest that ethnicity influences response to therapy.

\section{Fish oil}

Fish oil has been widely used in IgAN for more than 10 years. This is based on benefits shown in the largest RCT so far published, although other smaller trials have not supported this benefit. Fish oil is also preferred by some physicians because of its apparently favourable adverse effect profile, especially compared with corticosteroids and immunosuppressive agents

\section{Table 1. Treatment strategies for IgA nephropathy}

\section{A case of IgAN with:}

Normal renal function, $<0.5 \mathrm{~g} / \mathrm{d}$ proteinuria, non-visible with or without recurrent visible haematuria

Proteinuria $>0.5 \mathrm{~g} / \mathrm{d}$

GFR $>50 \mathrm{ml} / \mathrm{min}$ but proteinuria $>1 \mathrm{~g} / \mathrm{d}$ despite 3-6 months optimal BP control and RAS blockade

Nephrotic range proteinuria $(>3.5 \mathrm{~g} / \mathrm{d})$

Acute kidney injury

Acute kidney injury with crescentic IgAN

\section{Management}

No specific treatment; aim for blood pressure $<130 / 80 \mathrm{mmHg}$. Regular follow-up in primary care

Start ACEi or ARB; titrate to achieve target proteinuria $<1 \mathrm{~g} / \mathrm{d}$; aim for $\mathrm{BP}<130 / 80$ $\mathrm{mmHg}$ if proteinuria $<1 \mathrm{~g} / \mathrm{d}$ and under $125 / 75 \mathrm{mmHg}$ if proteinuria $>1 \mathrm{~g} / \mathrm{d}$

Consider six-month trial of corticosteroids

Treat as minimal-change disease with corticosteroids if this coexists on renal biopsy Supportive management

Treat as for renal vasculitis with cyclophosphamide and corticosteroids

$\mathrm{ACE}=$ angiotensin-converting enzyme inhibitor; $\mathrm{ARB}=$ angiotensin receptor blocker; $\mathrm{BP}=$ blood pressure; $\mathrm{GFR}=$ glomerular filtration rate; IgA = immunoglobulin $\mathrm{A} ;$ $\operatorname{IgAN}=\operatorname{IgA}$ nephropathy. 


\section{Tonsillectomy}

Tonsillectomy has been widely used in the management of IgAN, especially in Japan. This is based on the association of tonsillar and other upper respiratory infections with visible haematuria in IgAN, and the probable role of the tonsil as a producer of pathogenic IgA1, given that the tonsils are a rich source of $B$ cells, which could secrete pathogenic IgA. Although observational data indicate that tonsillectomy could reduce episodes of visible haematuria, it is less clear whether this strategy prevents later renal failure and, of course, the risks associated with tonsillectomy are not trivial. Thus, there is still no RCT evidence supporting a role for tonsillectomy.

\section{Nephrotic syndrome}

Nephrotic syndrome in IgAN was not uniformly responsive to corticosteroid therapy in the one available RCT. However, there was response in the minority of patients, with little or no light microscopic change, and it seems probable these are cases where IgAN and minimal-change nephrotic syndrome (the two most common glomerular diseases in young adults) have coincided. However, this does not extrapolate to justification for the use of corticosteroids in all cases of IgAN with nephrotic-range proteinuria.

\section{Acute kidney injury}

Acute kidney injury, often with visible haematuria, occurs in IgAN in two contexts, and a renal biopsy is required to differentiate them. In some patients, the biopsy finding is of acute tubular injury resulting from the tubular toxicity of haematuria. With supportive management, renal function usually spontaneously resolves as haematuria settles. The other context is crescentic IgAN with severe glomerular inflammation. Observational studies in this crescentic form of IgAN favour the use of cyclophosphamide and high-dose corticosteroids in regimens similar to those used for ANCA-positive renal vasculitis. However, the outcome is variable, which might relate to the extent of preexisting chronic renal injury.

\section{Conclusion}

The heterogeneity of the entity currently called IgAN has undoubtedly slowed progress in understanding this common glomerular disease. Further refinement of knowledge of disease mechanisms in IgAN is needed, despite substantial recent progress in understanding of the structural differences and pathogenicity of the IgA molecule.

Better definition of those individuals at high risk of progression using both clinical and pathological criteria is needed not only to improve prognostication and management for individual patients, but also to inform the design of future clinical trials. Even with such progress, the design of treatment trials in IgAN will still be challenging because of the prolonged follow-up needed to define outcome with confidence.

For the present, management of these patients remains centred around generic strategies in common with other slowly progressive kidney diseases, including reduction of proteinuria using RAS blockade and blood pressure control, with concomitant salt restriction.

\section{References}

1 Floege J. Recurrent IgA nephropathy after renal transplantation. Semin Nephrol 2004;24:287-91.

2 Boyd JK, Cheung CK, Molyneux K et al. An update on the pathogenesis and treatment of IgA nephropathy. Kidney Int 2012;81:833-43.

3 Bartosik LP, Lajoie G, Sugar L, Cattran DC. Predicting progression in IgA nephropathy. Am J Kidney Dis 2001;38:728-35.

4 Kanno Y, Okada H, Saruta T, Suzuki H. Blood pressure reduction associated with preservation of renal function in hypertensive patients with IgA nephropathy: a 3-year follow-up. Clin Nephrol 2000;54:360-5.

5 Reich HN, Troyanov S, Scholey JW, Cattran DC, Toronto Glomerulonephritis Registry. Remission of proteinuria improves prognosis in IgA nephropathy. J Am Soc Nephrol 2007;18:3177-83.

6 Cattran DC, Coppo R, Cook HT et al. The Oxford classification of IgA nephropathy: rationale, clinicopathological correlations, and classification. Kidney Int 2009;76:534-45.

7 Eitner F, Ackermann D, Hilgers R-D, Floege J. Supportive Versus Immunosuppressive Therapy of Progressive IgA nephropathy (STOP) IgAN trial: rationale and study protocol. J Nephrol 2008;21:284-9.

8 KDIGO. Clinical Practice Guidelines for Glomerulonephritis. Kidney Int Suppl 2012;2:139-274.

Address for correspondence: Prof J Feehally, The John Walls Renal Unit, Leicester General Hospital, Gwendolen Road, Leicester LE5 4PW.

Email: jf27@le.ac.uk 